\title{
Crank-Nicolson and Modified Crank-Nicolson Scheme for One Dimensional Parabolic Equation
}

\author{
Omowo Babajide Johnson ${ }^{1,}$, , Longe Idowu Oluwaseun ${ }^{2}$ \\ ${ }^{1}$ Department of Mathematics, Nasarawa State University, Keffi, Nigeria \\ ${ }^{2}$ Department of Statistics, Federal Polytechnic, Ile-Oluji, Nigeria
}

Email address:

johnsonomowo1@gmail.com (O. B. Johnson), longeseun@gmail.com (L. I. Oluwaseun)

${ }^{*}$ Corresponding author

\section{To cite this article:}

Omowo Babajide Johnson, Longe Idowu Oluwaseun. Crank-Nicolson and Modified Crank-Nicolson Scheme for One Dimensional Parabolic Equation. International Journal of Applied Mathematics and Theoretical Physics. Special Issue: Computational Mathematics.

Vol. 6, No. 3, 2020, pp. 35-40. doi: 10.11648/j.ijamtp.20200603.11

Received: May 28, 2020; Accepted: July 13, 2020; Published: August 13, 2020

\begin{abstract}
Partial differential equations are very important tools for mathematical modeling in some field like; physics, engineering and applied Mathematics. It's worth knowing that only few of this equation can be solved analytically and numerical method have been proven to perform exceedingly well in solving even difficult partial differential equations. Finite difference method is a popular numerical method which has been applied extensively to solve partial differential equations. A well known type of this method is the Classical Crank-Nicolson scheme which has been used by different researchers. In this work, we present a modified Crank-Nicolson scheme resulting from the modification of the classical Crank-Nicolson scheme to solve one dimensional parabolic equation. We apply both the Classical Crank-Nicolson scheme and the modified CrankNicolson scheme to solve one dimensional parabolic partial differential equation and investigate the results of the different schemes. The computation and results of the two schemes converges faster to the exact solution. It is shown that the modified Crank-Nicolson method is more efficient, reliable and better for solving Parabolic Partial differential equations since it requires less computational work. The method is stable and the convergence is fast when the results of the numerical examples where compared with the results from other existing classical scheme, we found that our method have better accuracy than those methods. Some numerical examples were considered to verify our results.
\end{abstract}

Keywords: Finite Difference Method, Parabolic Equation, Crank-Nicolson Scheme, Modified Crank-Nicolson Method, Stability

\section{Introduction}

Partial differential equations are the basis of many mathematical models of Physics, Chemistry, and Biology even in Finance. Approximating solutions of partial differential equations is very necessary and important, so as to investigate the predictions of the mathematical models, as exact solutions are usually difficult and uneasy to obtain. Problems involving time $t$ as one independent variable result most usually to parabolic equation, which are derives from the theory of heat conduction. Solutions of such problems can be obtained by numerical methods.

Many researchers have worked on the well-known parabolic partial differential equation (the one dimensional heat conduction equation) using various numerical methods but of all the numerical methods, finite difference methods are mostly used. There are many types of finite difference approximation used to solve the heat equation. We have the explicit and implicit finite difference methods and their modifications.

C. E. Abhulimen and B. J. Omowo [1] solved the heat diffusion problem using modified Crank-Nicolson method and compare the solution with the exact solutions. J. Crank and N. Philis [2] considered a practical method for numerical solution of partial differential equations of heat conduction type. E. C. DuFort and S. P. Frankel [4] modified the simple explicit scheme (FTCP) and proved that it is much more stable than the simple explicit case, enabling larger time steps to be used. S. E. Fadugba, et. al [6] applied Crank-Nicolson 
method to parabolic partial differential equation problems and demonstrated that the method is good for such problems. H. A. Isede [10] presented the Crank-Nicolson method being juxtaposed with the analytical solution applied to the diffusion equation.

J. Cooper [3], E. Kreyszig [11], G. D. Smith [13], and Williams. F. Ames [14] are very good and exhaustive texts on finite difference method among others.

Partial differential equations are problems involving rate of change of functions of several variables. For examples

a) Advectionequation: $\frac{\partial y}{\partial t}+\mathrm{v} \frac{\partial x}{\partial x}=0$

b) Heatequation: $\frac{\partial f}{\partial t}=\mathrm{D} \frac{\partial^{2} f}{\partial x^{2}}$

c) Poissonequation: $\frac{\partial^{2} f}{\partial x^{2}}-\frac{\partial^{2} f}{\partial y^{2}}=\mathrm{u}(\mathrm{x}, \mathrm{y})$

d) Waveequation: $\frac{\partial^{2} f}{\partial t^{2}}-\frac{\partial^{2} f}{\partial y^{2}}=0$

In the above equations, $x, y$ are space coordinates, $v$, $\mathrm{D}, \mathrm{c}$, are real positive constants and $\mathrm{t}, \mathrm{x}$ are often said to be time and space coordinates respectively. The general second order linear partial differential equation with two independent variables and one dependent variable is given by

$$
\mathrm{A} \frac{\partial^{2} f}{\partial x^{2}}+\mathrm{B} \frac{\partial^{2} f}{\partial x \partial y}+\mathrm{C} \frac{\partial^{2} f}{\partial y^{2}}+\mathrm{D}=0
$$

Here, A, B, C are functions of independent variables, and $\mathrm{x}$, $\mathrm{y}, \mathrm{D}$ are functions of $\mathrm{x}, \mathrm{y}, \mathrm{f}, \frac{\partial f}{\partial x}$ and $\frac{\partial f}{\partial y}$. It is important to note that for a parabolic partial differential equation to be parabolic, $b^{2}-4 a c=0$ is required. The one dimensional heat conduction equation of the form

$$
\frac{\partial f}{\partial t}=\frac{\partial^{2} f}{\partial x^{2}}
$$

Is a well known example of a parabolic partial differential equations. The solution of these equation is a temperature function $(\mathrm{x}, \mathrm{t})$ which is defined for values of $\mathrm{x}$ from 0 to 1 and for values of $t$ from 0 to $\infty$. The solution is not defined in a closed domain but advances in an open- ended region from initial values satisfying the prescribed boundary conditions.

\section{Finite Difference Method}

This is a method for solving partial differential equations. The procedure is simply by substituting the partial derivatives by difference equations in order to obtain the numerical solutions. There are different types of finite difference methods but we shall focus on the following in this work:

a) Crank-Nicolson Method and

b) Modified Crank-Nicolson Method

We shall use the following finite difference approximation as seen in Williams. F. Ames [14].

$$
\begin{gathered}
\frac{\partial f}{\partial t}=\frac{f_{i, j+1}-f_{i, j}}{k} \\
\frac{\partial f}{\partial t}=\frac{f_{i, j+1}-f_{i, j-1}}{2 k}
\end{gathered}
$$

$$
\frac{\partial^{2} f}{\partial x^{2}}=\frac{f_{i+1, j}-2 f_{i, j}+f_{i-1, j}}{h^{2}}
$$

and

$$
\frac{\partial^{2} f}{\partial x^{2}}=\frac{f_{i+1, j}-f_{i, j+1}-f_{i, j-1}+f_{i-1, j}}{h^{2}}
$$

\subsection{Derivation of Crank-Nicolson Scheme}

The classical Crank-Nicolson scheme is derived using the implicit scheme and the explicit finite difference scheme. Using finite difference approximation (3) that is,

$$
\begin{gathered}
\frac{\partial f}{\partial t}=\frac{f_{i, j+1}-f_{i, j}}{k} \text { and } \\
\frac{\partial^{2} f}{\partial x^{2}}=\frac{f_{i-1, j}-2 f_{i, j}+f_{i+1, j}}{h^{2}}
\end{gathered}
$$

Then equation (2) is analogue to

$$
\frac{1}{k}\left(f_{i, j+1}-f_{\mathrm{i}, \mathrm{j}}\right)=\frac{1}{h^{2}}\left(f_{i-1, j}-2 f_{i, j}+f_{i+1, j}\right.
$$

In equation (4), the function values along the $j^{\text {th }}$ row only is used. The $(j+1)^{\text {th }}$ approximation for $f_{x x}$ in (2) is given by

$$
\frac{1}{k}\left(f_{i, j+1}-f_{i, j}\right)=\frac{f_{i-1, j+1}-2 f_{i, j+1}+f_{i+1, j+1}}{h^{2}}
$$

Taking average of the $j^{\text {th }}$ and $(j+1)^{\text {th }}$ rows we have

$$
\begin{gathered}
\frac{f_{i, j+1}-f_{i, j}}{k}=\frac{1}{2}\left[\frac{f_{i-1, j}-2 f_{i, j}+f_{i+1, j}}{h^{2}}\right]+ \\
\frac{1}{2}\left[\frac{f_{i-1, j+1}-2 f_{i, j+1}+f_{i+1, j+1}}{h^{2}}\right]
\end{gathered}
$$

(2) is therefore replaced with

$$
\begin{gathered}
2\left(f_{i, j+1}-f_{\mathrm{i}, j}\right)=\frac{k}{h^{2}}\left(f_{i-1, j}-2 f_{i, j}\right. \\
\left.+f_{i+1, j}+f_{i-1, j+1}-2 f_{i, j+1}+f_{i+1, j+1}\right)
\end{gathered}
$$

Let $\frac{k}{h^{2}}=r$, on solving we get

$$
\begin{aligned}
-\mathrm{r} f_{i-1, j+1}+ & (2+2 r) f_{i, j+1}-r f_{i+1, j+1}=\mathrm{r} f_{i-1, j}+ \\
& (2-2 r) f_{i, j}+r f_{i+1, j}
\end{aligned}
$$

which can be written as

$$
\begin{gathered}
2(1+\mathrm{r}) f_{i, j+1}-r\left[f_{i-1, j+1}+f_{i+1, j+1}\right]=2(1-\mathrm{r}) f_{i, j}+r\left[f_{i-1, j}+\right. \\
\left.f_{i+1, j}\right]
\end{gathered}
$$

Equation (6) is the Crank-Nicolson scheme.

\subsection{Derivation of Modified Crank-Nicolson Scheme}

For the derivation of the Modified Crank-Nicolson scheme, L. H. S of (5) is replaced by $\frac{f_{i, j}-f_{i, j-1}}{k}$ and the $(j+1)^{\text {th }}$ row becomes $(j-1)^{\text {th }}$ row, then the finite difference analogue for (2) becomes

$$
\begin{gathered}
2\left(f_{i, j}-f_{i, j-1}\right)=\frac{k}{h^{2}}\left(f_{i+1, j-1}-2 f_{i, j-1}+f_{i-1, j-1}+f_{i+1, j}-\right. \\
\left.2 f_{i, j}+f_{i-1, j}\right)
\end{gathered}
$$


Put $r=\frac{k}{h^{2}}$, on solving we have

$$
\begin{gathered}
-2 r f_{i, j-1}+2 f_{i, j-1}+r f_{i+1, j-1}+r f_{i-1, j-1}=-r f_{i+1, j}+ \\
2 f_{i, j}+2 r f_{i, j}-r f_{i-1, j}
\end{gathered}
$$

which can be written as

$$
\begin{gathered}
2(1+r) f_{i, j}-r\left(f_{i+1, j}+f_{i-1, j}\right)=2(1-r) f_{i, j-1}+ \\
r\left(f_{i+1, j-1}+f_{i-1, j-1}\right)
\end{gathered}
$$

(8) is our modified Crank-Nicolson scheme, which allows the computation to start from $i=1, j=1$ different from the classical Crank-Nicolson whose computation starts from $i=1$, $j=0$.

\subsection{Stability of Crank-Nicolson Scheme}

Consider the equation (6) given as

$$
\begin{gathered}
2(1+r) f_{i, j+1}-r\left[f_{i-1, j+1}+f_{i+1, j+1}\right]=2(1-r) f_{i, j}+r\left[f_{i-1, j}+\right. \\
\left.f_{i+1, j}\right]
\end{gathered}
$$

worse case solution is given as

$$
f_{i, j}=\alpha^{j}(-1)^{i}(\text { high frequency } x \text {-oscillations in index } i)
$$

substituting (9) into (7) to get

$$
\begin{gathered}
2(1+r) \alpha^{j+1}(-1)^{i}-r \alpha^{j+1}(-1)^{i-1}\left[(-1)^{i-1}+\right. \\
\left.(-1)^{i+1}\right]=2(1-r) \alpha^{j}(-1)^{i}+\alpha r^{j}(-1)^{i-1}+\alpha r^{j}(-1)^{i+1} \\
r[-\alpha(-1)-1+2(1+r)-\alpha(-1)+1]=r(-1)-1+ \\
2(1-r)+r(-1)+1
\end{gathered}
$$

which gives

$$
\alpha=\frac{[1-2 r]}{[1+2 r]}
$$

then

$$
\left|\alpha=\frac{1-2 r}{1+2 r}\right| \rightarrow|\alpha|<1, \forall \alpha>0
$$

The stability of modified Crank-Nicolson method is derived using the same approach in C. E. Abhulimen and B. J. Omowo [1]. The modified Crank-Nicolson method is unconditionally stable and has higher order accuracy. The price of solving a tri-diagonal system at each step is worth paying since the method allows large step sizes.

\section{Numerical Examples}

This section presents some numerical examples, where we compare the computation results of both the classical CrankNicolson method and the modified Crank-Nicolson method.

Example1:

Derive the exact solution of the partial differential equation and confirm the results using Crank-Nicolson scheme and Modified Crank-Nicolson Scheme.

$$
\left\{\begin{array}{c}
\frac{1}{2} \frac{\partial^{2} f}{\partial x^{2}}=\frac{\partial f}{\partial t}, 0 \leq x \leq 4 \\
\operatorname{with} f(x, 0)=x(4-x) \\
\operatorname{and} f(0, t)=0=f(4, t)
\end{array}\right.
$$

Solution:

The exact solution of example 1 above is derived in C. E. Abhulimen and B. J. Omowo [1] and is given by

$$
f=\frac{128}{\pi^{3}} \sin \left(\frac{n \pi}{4}\right) x e^{-\left(\frac{n^{2} \pi^{2}}{32}\right) t}
$$

from where the analytical solutions for $f_{1}, f_{2}$ and $f_{3}$ are $2.1356,3.03258$ and 2.1356 respectively.

Now, let solve example 1 above using Crank-Nicolson scheme as follows:

Crank-Nicolson scheme is given by (6) at $i=1, \mathrm{j}=0$, we have

$$
\begin{gathered}
3 f_{1.1}-0.5 f_{0,1}-0.5 f_{2,1}=f_{1,0}+0.5 f_{2,0} \\
3 f_{1,1}-0.5 f_{2,1}=5
\end{gathered}
$$

at $2 \leq i \leq 3, \mathrm{j}=0$, we have the following

$$
\begin{aligned}
& 3 f_{2,1}-0.5 f_{1,1}-0.5 f_{3,1}=7 \\
& 3 f_{3,1}-0.5 f_{2,1}-0.5 f_{4,1}=5
\end{aligned}
$$

solving equations (13)-(15) we have that $f_{1,1}=2.1765, f_{2,1}=3.0588$ and $f_{3,1}=2.1765$

Now, we move to the next step at $1 \leq i \leq 3, j=1$, we have the following equations

$$
\begin{gathered}
3 f_{1,2}-0.5 f_{2,2}=3.7059 \\
3 f_{2,2}-0.5 f_{1,2}-0.5 f_{3,2}=5.2353 \\
3 f_{3,2}-0.5 f_{2,2}=3.7059
\end{gathered}
$$

solving the equations (16)-(18) we have $f_{1,2}=1.6159, f_{2,2}=2.287, f_{3,2}=1.6159$ for next step, at $1 \leq i \leq 3, j=2$, we have the following equations

$$
\begin{aligned}
& 3 f_{1,3}-0.5 f_{2,3}=2.75775 \\
& 3 f_{2,3}-0.5 f_{1,3}-0.5 f_{3,3}=3.8996 \\
& 3 f_{3,3}-0.5 f_{2,3}-0.5 f_{4,3}=2.75775
\end{aligned}
$$

Solving equations (19)-(21) we get $f_{1,3}=1,2027, f_{2,3}=$ $1.7008, f_{3,3}=1.202$.

We shall now apply the Modified Crank-Nicolson scheme to solve the same problem (example 1) as follows:

Modified Crank-Nicolson scheme is given by (7) at $1 \leq i \leq 3, j=1$ (firststep) we have the following equations

$$
\begin{gathered}
3 f_{1,1}-0.5 f_{2,1}=5 \\
3 f_{2,1}-0.5 f_{1,1}-0.5 f_{3,1}=7 \\
3 f_{2,1}-0.5 f_{1,1}-0.5 f_{3,1}=7(23) 3 f_{3,1}-0.5 f_{2,1}=5
\end{gathered}
$$


solving equations (22)-(24) we have that

$$
f_{1,1}=2.1765, f_{2,1}=3.0588 \text { and } f_{3,1}=2.1765
$$

next step is at $1 \leq i \leq 3, j=2$, we have

$$
\begin{gathered}
3 f_{1,2}-0.5 f_{2,2}=3.7059 \\
3 f_{2,2}-0.5 f_{1,2}-0.5 f_{3,2}=5.2353 \\
3 f_{3,2}-0.5 f_{2,2}=3.7059
\end{gathered}
$$

Solving the equations (25)-(27) we have

$$
f_{1,2}=1.6159, f_{2,2}=2.287, f_{3,2}=1.6159
$$

next step is at $i=1, j=3$

$$
3 f_{1,3}-0.5 f_{2,3}=2.75775
$$

at $i=2, \mathrm{j}=3$

$$
3 f_{2,3}-0.5 f_{1,3}-0.5 f_{3,3}=3.8996
$$

at $i=3, \mathrm{j}=3$

$$
3 f_{3,3}-0.5 f_{2,3}-0.5 f_{4,3}=2.75775
$$

solving equations (28)-(30) we get $f_{1,3}=1,2027, f_{2,3}=$ $1.7008, f_{3,3}=1.2027$.

Example2

Solve the parabolic equation using Crank-Nicolson scheme and Modified Crank-Nicolson scheme

$$
\begin{gathered}
\frac{\partial^{2} f}{\partial x^{2}}-\frac{\partial f}{\partial t}=0 \\
\text { with } f(x, 0)=x^{2}\left(25-x^{2}\right) \\
\text { and } f(0, t)=0=f(5, t)
\end{gathered}
$$

where $\mathrm{h}=1, \mathrm{k}=\frac{1}{2}$,

solution:

$$
\begin{gathered}
r=\frac{k}{h^{2}}=\frac{1}{2}, \text { now } f(x, 0)=x^{2}\left(25-x^{2}\right), \\
f(1,0)=1(25-1)=24, f(2,0)=4(25-4)=84 \\
f(3,0)=144, f(4,0)=144, \text { and } f(5,0)=0 \text { also, } \\
f(0, t)=f(5, t)
\end{gathered}
$$

using Crank-Nicolson scheme (6) at $i=1, j=0$ we have

$$
\begin{gathered}
2\left(1+\frac{1}{2}\right) f_{1,1}-\frac{1}{2} f_{2,1}-\frac{1}{2} f_{0,1}=f_{1,0}-\frac{1}{2} f_{2,0}-\frac{1}{2} f_{0,0} \\
3 f_{1.1}-0.5 f_{0,1}-0.5 f_{2,1}=f_{1,0}+0.5 f_{2,0}+0.5 f_{0,0} \\
3 f_{1,1}-0.5 f_{2,1}=66
\end{gathered}
$$

Solving for $2 \leq i \leq 4$ at $j=0$, using (6) we have set of equations and together with (32) forms a tri-diagonal matrix of the form

$$
\left[\begin{array}{cccc}
3 & -0.5 & 0 & 0 \\
-0.5 & 3 & -0.5 & 0 \\
0 & -0.5 & 3 & -0.5 \\
0 & 0 & -0.5 & 3
\end{array}\right]\left[\begin{array}{l}
f_{1,1} \\
f_{2,1} \\
f_{3,1} \\
f_{4,1}
\end{array}\right]=\left[\begin{array}{c}
66 \\
168 \\
258 \\
216
\end{array}\right]
$$

Solving the matrix equation above gives $f_{1,1}=35.5055, f_{2,1}=81.0328, f_{3,1}=114.6913, f_{4,1}=$

91.1152 for the next step, we have at $1 \leq i \leq 4$, for $j=1$ and using (6), gives a matrix equation of the form

$$
\left[\begin{array}{cccc}
3 & -0.5 & 0 & 0 \\
-0.5 & 3 & -0.5 & 0 \\
0 & -0.5 & 3 & -0.5 \\
0 & 0 & -0.5 & 3
\end{array}\right]\left[\begin{array}{l}
f_{1,2} \\
f_{2,2} \\
f_{3,2} \\
f_{4,2}
\end{array}\right]=\left[\begin{array}{c}
76.0219 \\
156.1312 \\
200.7653 \\
148.4609
\end{array}\right]
$$

Solving the above matrix gives $f_{1,2}=37.5544, f_{2,2}=$ $73.2828, f_{3,2}=89.8801, f_{4,2}=64.4670$ and similarly for the next step at $1 \leq \mathrm{i} \leq 4$ for $\mathrm{j}=2$ gives a matrix equation with the following results $f_{1,3}=35.3118, f_{2,3}=63.4794, f_{3,3}=$ 71.5643, $f_{4,3}=48.3964$

Using Modified Crank-Nicolson scheme:

Instead of starting with $\mathrm{i}=1$ and $\mathrm{j}=0$ we start at $\mathrm{i}=1, \mathrm{j}=1$ therefore using (8) we have

$$
\begin{gathered}
2\left(1+\frac{1}{2}\right) f_{1,1}-\frac{1}{2} f_{2,1}-\frac{1}{2} f_{0,1}=2\left(1-\frac{1}{2}\right) f_{1,0}+\frac{1}{2} f_{2,0}+\frac{1}{2} f_{0,0} \\
3 f_{1.1}-0.5 f_{0,1}-0.5 f_{2,1}=f_{1,0}+0.5 f_{2,0}+0.5 f_{0,0} \\
3 f_{1,1}-0.5 f_{2,1}=66
\end{gathered}
$$

On solving for $i=2 \leq i \leq 4$ and $j=1$ we get a system of matrix equation in the form

$$
\left[\begin{array}{cccc}
3 & -0.5 & 0 & 0 \\
-0.5 & 3 & -0.5 & 0 \\
0 & -0.5 & 3 & -0.5 \\
0 & 0 & -0.5 & 3
\end{array}\right]\left[\begin{array}{c}
f_{1,1} \\
f_{2,1} \\
f_{3,1} \\
f_{4,1}
\end{array}\right]=\left[\begin{array}{c}
66 \\
168 \\
258 \\
216
\end{array}\right]
$$

solving the matrix above gives $f_{1,1}=35.5055, f_{2,1}=$ 81.0328, $f_{3,1}=114.6913, f_{4,1}=91.1152$ for the next step we have at $1 \leq i \leq 4$ and $j=2$ gives the following matrix system

$$
\left[\begin{array}{cccc}
3 & -0.5 & 0 & 0 \\
-0.5 & 3 & -0.5 & 0 \\
0 & -0.5 & 3 & -0.5 \\
0 & 0 & -0.5 & 3
\end{array}\right]\left[\begin{array}{l}
f_{1,2} \\
f_{2,2} \\
f_{3,2} \\
f_{4,2}
\end{array}\right]=\left[\begin{array}{c}
76.0219 \\
156.1312 \\
200.7653 \\
148.4609
\end{array}\right]
$$

on solving the above matrix we have the following results $f_{1,2}=37.5544, f_{2,2}=73.2828, f_{3,2}=89.8801, f_{4,2}=$ 64.4670 which is the same results obtained using CrankNicolson method for $1 \leq i \leq 4$ at $\mathrm{j}=1$. For $1 \leq i \leq 4$, at $\mathrm{j}=3$ we have the following solutions $f_{1,3}=35.3118, f_{2,3}=$ $63.4794, f_{3,3}=71.5643, f_{4,3}=48.3964$ which is the same results obtain with Crank-Nicolson method for $1 \leq i \leq 4$ at $\mathrm{j}=2$. 
Example3:

Solve the partial differential equation using modified Crank-Nicolson scheme:

$$
\begin{gathered}
\frac{\partial^{2} f}{\partial x^{2}}=\frac{\partial f}{\partial t} 0 \leq x \leq 1 \\
\text { with } f(x, 0)=100 \sin \pi x \\
\text { and } f(0, t)=0=f(1, t)
\end{gathered}
$$

Solution:

Here, we use $h=0.1$ and $k=0.004$ then $r=\frac{k}{h^{2}}=\frac{2}{5}<\frac{1}{2}$. Using (8), we have at $i=1, j=1$

$$
\begin{gathered}
2\left(1+\frac{2}{5}\right) f_{1,1}-\frac{2}{5} f_{2,1}-\frac{2}{5} f_{0,1}=2\left(1-\frac{2}{5}\right) f_{1,0}+\frac{2}{5} f_{2,0}+\frac{2}{5} f_{0,0} \\
2.8 f_{1.1}-0.4 f_{0,1}-0.4 f_{2,1}=1.2 f_{1,0}+0.4 f_{2,0}+0.4 f_{0,0} \\
2.8 f_{1,1}-0.4 f_{2,1}=60.5934
\end{gathered}
$$

at $i=2, \mathrm{j}=1$ we have

$$
\begin{aligned}
& 2.8 f_{2.1}-0.4 f_{3,1}-0.4 f_{1,1}=1.2 f_{2,0}+0.4 f_{3,0}+0.4 f_{1,0} \\
& 2.8 f_{2,1}-0.4 f_{3,1}-0.4 f_{1,1}=115.2556
\end{aligned}
$$

at $i=3, \mathrm{j}=1$

$$
2.8 f_{3,1}-0.4 f_{4,1}-0.4 f_{2,1}=158.6357
$$

at $i=4, \mathrm{j}=1$

$$
2.8 f_{4,1}-0.4 f_{5,1}-0.4 f_{3,1}=186.4875
$$

at $i=5, \mathrm{j}=1$

$$
2.8 f_{5,1}-0.4 f_{6,1}-0.4 f_{4,1}=196.0846
$$

at $i=6, \mathrm{j}=1$

$$
2.8 f_{6,1}-0.4 f_{7,1}-0.4 f_{5,1}=186.4875
$$

at $i=7, \mathrm{j}=1$

$$
2.8 f_{7,1}-0.4 f_{8,1}-0.4 f_{6,1}=158.6357
$$

at $i=8, \mathrm{j}=1$

$$
2.8 f_{8,1}-0.4 f_{9,1}-0.4 f_{7,1}=115.2556
$$

at $i=9, \mathrm{j}=1$

$$
2.8 f_{9,1}-0.4 f_{8,1}=60.4934
$$

Just as above, we have a matrix system represented as

$$
\left[\begin{array}{ccccccccc}
2.8 & -0.4 & 0 & 0 & 0 & 0 & 0 & 0 & 0 \\
-0.4 & 2.8 & -0.4 & 0 & 0 & 0 & 0 & 0 & 0 \\
0 & -0.4 & 2.8 & -0.4 & 0 & 0 & 0 & 0 & 0 \\
0 & 0 & -0.4 & 2.8 & -0.4 & 0 & 0 & 0 & 0 \\
0 & 0 & 0 & -0.4 & 2.8 & -0.4 & 0 & 0 & 0 \\
0 & 0 & 0 & 0 & -0.4 & 2.8 & -0.4 & 0 & 0 \\
0 & 0 & 0 & 0 & 0 & -0.4 & 2.8 & -0.4 & 0 \\
0 & 0 & 0 & 0 & 0 & 0 & -0.4 & 2.8 & -0.4 \\
0 & 0 & 0 & 0 & 0 & 0 & 0 & -0.4 & 2.8
\end{array}\right]\left[\begin{array}{l}
f_{1,1} \\
f_{2,1} \\
f_{3,1} \\
f_{4,1} \\
f_{5,1} \\
f_{6,1} \\
f_{7,1} \\
f_{8,1} \\
f_{9,1}
\end{array}\right]
$$

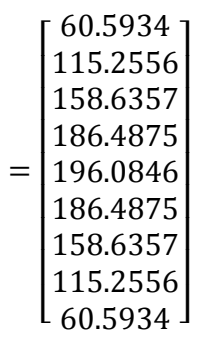

which is atri-diagonal system.

\section{Discussion}

The modified Crank-Nicolson method from observation is fast and effective since, the value at $i=1, j=0$ for CrankNicolson method is the same at $i=1, j=1$ will give using the modified Crank-Nicolson method, hence no need for computing at $i=1, j=0$ instead we start from $i=1, j=1$, which reduces computational stress and makes convergent faster than the former.

\section{Conclusion}

In this work, modified Crank-Nicolson method has been successfully compared with the classical Crank-Nicolson method by applying it to solve problems on parabolic partial differential equations (Heat conduction problems). We solved some numerical examples to illustrate how effective the scheme is by comparing it with the classical Crank-Nicolson scheme. In the process, it was discovered that the modified Crank-Nicolson scheme is efficient and reduces computational stress.

We remark that, the modified Crank-Nicolson scheme is very quick, reliable, unconditionally stable and faster in application (without giving up accuracy) when compared with the other existing methods stated in the literature.

\section{References}

[1] C. E Abhulimen and B. J Omowo, Modified Crank-Nicolson Method for solving one dimensional Parabolic equations. IOSR Journal of Mathematics, Volume15, Issue 6 series 3 (2019) pg 60-66.

[2] J. Crank and N. Philis, A practical method for Numerical Evaluation of solution of partial differential equation of heat conduction type. Proc. Camb. Phil. Soc. 1 (1996), 50-57.

[3] J. Cooper, Introduction to Partial differential Equation with Matlab, Boston, 1958.

[4] E. C DuFort and S. P Franel, Conditions in Numerical Treatment of Partial differential equations. Math. Comput. 7 (43) (1953) 135-152.

[5] Emenogu George Ndubueze and Oko Nlia, Solutions of parabolic partial differential equations by finite difference methods. Journal of Applied Mathematics 8 (2), 2015, 88-102.

[6] S. E Fadugba, O. H Edogbanya, S. C Zelibe, Crank-Nicolson method for solving parabolic partial differential equations. International Journal of Applied Mathematics and Modeling IJA2M, vol 1, (2013) nos 3pp 8-23. 
[7] A. Fallahzadeh and K. Shakibi, A method to solve Convection-diffusion equation based on homotopy analysis method. Journal of Interpolation and Approximation in scientific computing 1, (2015) pp1-8.

[8] Febi Sanjaya and Sudi Mungkasi, A simple but accurate explicit finite difference method for Advection-diffusion equation, Journal of Phy, Conference series 909, (2017).

[9] W. Gerald Recktennwald, Finite difference Approximation to the heat equation, Mathematical Method, 8 (34) 2004 pp747760 .

[10] H. A Isede, Several examples of Crank-Nicolson method for parabolic partial differential equations. Academia Journal of Scientific Research 1 (4) (2013), 06 3-068.
[11] E. Kreyszig, Advanced Engineering Mathematics, USA, John Wiley and Sons, pp 861-865.

[12] Neethu Fernandes and Rakhi B, An overview of CrankNicolson method to solve parabolic partial differential equations. International Journal of Scientific Research, vol 7, issue $12,1074-1086$.

[13] G. D Smith, Numerical Solution of Partial differential equations: Finite difference methods, Oxford Applied Mathematics and Computing science series, Oxford University press, Third edition, 1985.

[14] Williams F. Ames, Numerical Methods for Partial differential Equations, Academic press, Inc, Third edition, 1992. 